\title{
Synergistic effects of ginsenoside Rg3 and cyclophosphamine on tumor growth and angiogenesis in lung cancer
}

Li Hang ${ }^{1 *}$, Wang Jun ${ }^{2}$, Guan Yanfeng ${ }^{3}$, Yang Wenjun ${ }^{4}$ Aiying ${ }^{1}$ Suo Aiying ${ }^{1}$, Chu Meihua ${ }^{1}$, Wang Chenyu ${ }^{1}$, Jiang Jing ${ }^{1}$, Zhai Shengping ${ }^{1}$ and Mao Qishan ${ }^{5}$

${ }^{1}$ Department of Respiratory Medicine, Yantai Yuhuangding Hospital, Yantai City, China.

${ }^{2}$ Department of Respiratory Medicine, Lai Zhou People's Hospital, Yantai City, Shandong Province, China.

${ }^{3}$ Medical Department, Haigang Hospital of Yantai, Yantai City, Shandong Province, China.

${ }^{4}$ Neurosurgery Department, Yantai Yuhuangding Hospital, Yantai City, China.

${ }^{5}$ Medical Department, Yantai Yuhuangding Hospital, Yantai City, China.

Accepted 13 July, 2011

\begin{abstract}
To evaluate the effectiveness of ginsenoside Rg3 alone or in combination with cyclophosphamide (CPA) on tumor growth and angiogenesis in human lung cancer, 54 female athymic mice were transplanted with lung cancer cells (A549) which then were randomly divided into 4 groups: Ginsenoside Rg3 group, CPA group, ginsenoside Rg3 plus CPA group and control group. Ginsenoside $\mathrm{Rg} 3$ of $3.0 \mathrm{mg} / \mathrm{kg}$ (once/day for 10 days) and CPA of $20.0 \mathrm{mg} / \mathrm{kg}$ (once/day for 10 days) were intraperitoneally given to mice for consecutive 10 days. Seven mice selected from each group were sacrificed 18 days later. The survival time of the remaining 7 mice in each group was recorded. The life elongation rate, proliferating cell nuclear antigen labeling index (PCNALI), expression of vascular endothelial cell growth factor (VEGF) and microvessel density (MVD) in the tumor tissues were evaluated. The quality of life of mice with administration of ginsenoside Rg3 alone or ginsenoside Rg3 plus CPA were better with longer survival time, when compared with other groups. The PCNALI, MVD and VEGF expression in mice of the treated groups were significantly lowered when compared with that of the control group. Additionally, the MVD of mice in groups with treatment of ginsenoside Rg3 alone or ginsenoside Rg3 plus CPA were lower than that in the CPA group. Tumor growth and angiogenesis in lung cancer were profoundly inhibited by ginsenoside Rg3 alone or in combination with CPA. The synergistic anticancer effects of ginsenoside Rg3 and CPA improved the survival time in lung cancer.
\end{abstract}

Key words: Ginseng, cyclophosphamide, angiogenesis, lung cancer.

\section{INTRODUCTION}

With the development of Chinese herbal medicine, more and more researchers are interested in the exploitation of novel anticancer components in herbs. Ginsenoside Rg3, the major active component of ginseng has high

*Corresponding author. E-mail: fengqiaoyebo@163.com . Tel: +86535 6691999-83202. Fax: +86535 6691999-83202.

Abbreviations: CPA, Cyclophosphamide; VEGF, vascular endothelial cell growth factor; PCNALI, proliferating cell nuclear antigen labeling index; MVD, microvessel density. anticancer activity. Studies have indicated its suppressive effects on the angiogenesis in Lewis cancer (Lee et al., 2009), breast cancer (Zhang et al., 2008) and on invasion and metastasis of intestinal adenocarcinomas and B16 melenoma (Liu et al., 2004; Mochizuki et al., 1995; lishi et al., 2004) as well as proliferation of prostate cancer cells (Kim et al., 2004; Keum et al., 2003). Recently, evidence has disclosed the therapeutic effects of continuous low dose chemotherapy combined with other inhibitors of angiogenesis (Hanahan et al., 2000; Klement et al., 2000). The results demonstrated that this method was more effective in the suppression of tumor growth than conventional therapy. However, the effects of this method 
on lung cancer remain to be evaluated.

In this study, we aimed to evaluate the effectiveness of ginsenoside $\mathrm{Rg} 3$ alone or in combination with cyclophosphamide (CPA) on the tumor growth and angiogenesis in human lung cancer.

\section{MATERIALS AND METHODS}

Ginsenoside Rg3, provided by the Department of Basic Medicine of Shandong University, was extracted from American ginseng with $\geq 99.5 \%$ of purity quotient. Six-week old female athymic mice were purchased from the department of Experimental Animal Center of Shandong University. The mouse monoclonal antibodies specific to CD31, vascular endothelial cell growth factor (VEGF) and proliferating cell nuclear antigen (PCNA) were purchased from Santa Cruz Co. (Santa Cruze, USA).

Human lung cancer cell line A549 was obtained from the Department of Basic Medicine of Shandong University and cultured in RPMI1640 supplemented with $10 \%$ fetal bovine serum. 28 female athymic mice were subcutaneously transplanted with $0.2 \mathrm{ml}$ $\left(1 \times 10^{7} / \mathrm{ml}\right)$ A549 cell suspension in the armpit of right anterior limb. These mice were randomly divided into 4 groups ( $n=14$ for each): Ginsenoside Rg3 group, CPA group, ginsenoside Rg3 plus CPA group and control group. Ginsenoside $\mathrm{Rg} 3$ was intraperitoneally administrated at a dose of $3.0 \mathrm{mg} / \mathrm{kg}$ (once/day for 10 days) and CPA of $20.0 \mathrm{mg} / \mathrm{kg}$ (once/day for 10 days). The mice in the control group received saline. Seven mice selected from each group were sacrificed 18 days later. The survival time of the remaining 7 mice in each group was recorded. During the experiment, the survival time was recorded within forty days after tumor transplantation. Additionally, the life elongation rate was analyzed. Tumor tissues were fixed immediately in $10 \%$ paraformaldehyde and embedded in paraffin. Proliferative activity of lung cancer cells was evaluated by immunohistochemical staining with mouse monoclonal PCNA antibody (1:100) and the number of positive cells was counted at $\times 400$ magnifications. Subsequently, proliferating cell nuclear antigen labeling index (PCNALI) was calculated.

Vascularization was expressed by MVD (micro-vascular density). The micro-vascular was evaluated by immunostaining of endothelium with mouse monoclonal CD31-PECAM antibody (1:100). The number of CD31 immunostained blood vessels was quantified at $\times 200$ magnification. The values from five different fields were averaged and presented as mean \pm standard deviation.

VEGF positive cells were stained with yellow brown in the cytoplasm. The intensity of the immunoreactive reaction were graded as 0 , no immunoreactivity; 1 , weak intensity; 2, moderate intensity; 3 , strong intensity. Positive percentage were graded as 0 , $<10 \% ; 1,10$ to $24 \%$; 2,25 to $49 \% ; 3,50$ to $75 \%$; $4 \geq 75$. After adding the scores of intensity and positive percentage, we rescaled to score 0 to 1 as negative (-), 2 to 3 as weak expression (1+), 4 to 5 as moderate expression (2+) and above 5 as strong expression $(3+)$, and the immunoreactivity was scored semi quantitatively as the intensity of the immunoreactive reaction and positive percent of tumor cells.

\section{Statistical analysis}

The results were expressed with mean \pm standard deviation. Statistical analysis was performed by LSD test and one way analysis of variance for multiple comparisons in evaluation of tumor weight and number of living day. Nemenyi test for two comparisons and Kruskal Wallis test for multiple comparisons were performed in the analysis of PCNALI, MVD (micro-vascular density) and VEGF. A value of $P<0.05$ was considered as statistically significant.

\section{RESULTS}

\section{Effects of ginsenoside Rg3 and CPA on life elongation}

40 days after tumor transplantation, the number of mice that survived in ginsenoside Rg3, CPA, ginsenoside Rg3 plus CPA and control groups was 3, 2, 3 and 0 , respectively. The survival time of mice with lung cancer in the ginsenoside Rg3, CPA and ginsenoside Rg3 plus CPA groups was longer than in the control group and the life elongation rate reached $98.2 \%$ in the mice of ginsenoside Rg3 plus CPA group (Table 1). The results demonstrate that ginsenoside $\mathrm{Rg} 3$ prolonged survival time of mice with lung cancer. Furthermore, the ginsenoside Rg3 and CPA conferred the synergistic therapeutic effects on the lung cancer.

\section{Effects of ginsenoside Rg3 and CPA on tumor cell proliferation and angiogenesis}

Table 2 and Figure 1 show that the number of PCNA positive cells and PCNALI in the treated groups was lower than that in the control group. These findings show that the ginsenoside Rg3, CPA or ginsenoside Rg3 in combination with CPA could inhibit the proliferation of A549 cells which leads to the suppression of tumor growth.

The density of blood vessels in tumor tissues was assessed by CD31-PECAM immunostaining. The MVD in the ginsenoside Rg3, CPA and ginsenoside Rg3 plus CPA groups was significantly lowered when compared with control group. Furthermore, in the ginsenoside Rg3 and ginsenoside Rg3 plus CPA groups, it was lower than CPA group (Table 2 and Figure 2). VEGF positive cells found in the ginsenoside Rg3, CPA and ginsenoside Rg3 plus CPA groups were markedly decreased when compared with control group, and there was no significant difference among the treated groups (Table 3 and Figure 3). The results show that ginsenoside Rg3 inhibited tumor angiogenesis and the angiosuppressive effects of ginsenoside $\mathrm{Rg} 3$ were improved by CPA.

\section{DISCUSSION}

Chemotherapy is the treatment of choice for a variety of malignant tumors, which together with surgery and radiotherapy constitute the traditional therapeutic methods for cancer. Although, chemotherapy is effective in combating against the tumor growth and metastasis, the mortality of cancer remains high. For example, 5-year survival rate of lung cancer remains low after conventional treatment (Davis et al., 2004). Thus, numerous studies have been conducted to explore novel therapies for cancers (Nguyen et al., 1998). Anti-angiogenesis is a promising 
Table 1. Effect of ginsenoside Rg3 and CPA on life elongation of mice.

\begin{tabular}{lccc}
\hline Group & Number of mice & Living day & Life elongation rate (\%) \\
\hline Control & 7 & $13.59 \pm 6.21$ & - \\
Rg3 & 7 & $23.81 \pm 9.22^{\star *}$ & 74.5 \\
CPA & 7 & $25.89 \pm 10.08^{\star \star}$ & 90.2 \\
CPA+Rg3 & 7 & $27.22 \pm 8.11^{\star *}$ & 98.2 \\
\hline
\end{tabular}

${ }^{*} P<0.05,{ }^{* *} P<0.01$ vs. control group.

Table 2. Inhibitory effects of ginsenoside Rg3 and CPA on tumor cell proliferation and angiogenesis (PCNALI and MVD in different groups).

\begin{tabular}{lcc}
\hline Group & PCNALI (\%) & Number of vessel (MVD) \\
\hline Control & $69.1 \pm 10.7$ & $28.3 \pm 3.1$ \\
Rg3 & $53.5 \pm 14.9^{*}$ & $23.0 \pm 2.64^{* *}$ \\
CPA & $43.1 \pm 12.3^{* *}$ & $18.9 \pm 2.14^{* *}$ \\
CPA+Rg3 & $35.5 \pm 12.7^{* *}$ & $10.8 \pm 2.88^{* *}$ \\
\hline
\end{tabular}

${ }^{*} P<0.05,{ }^{* *} P<0.01$ vs control group. PCNALI, proliferating cell nuclear antigen labeling index; MVD, microvessel density.

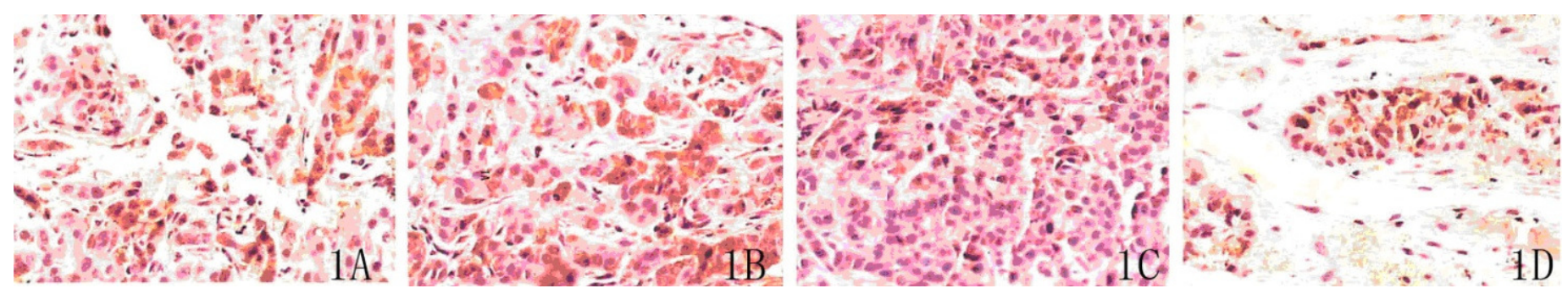

Figure 1. Immunohistochemical staining of PCNA. PCNA positive cells have yellow brown granules in nucleus. A: Control group; B: ginsenoside Rg3 group; C: CPA group; D: ginsenoside Rg3 plus CPA group (magnification $\times 200$ ).
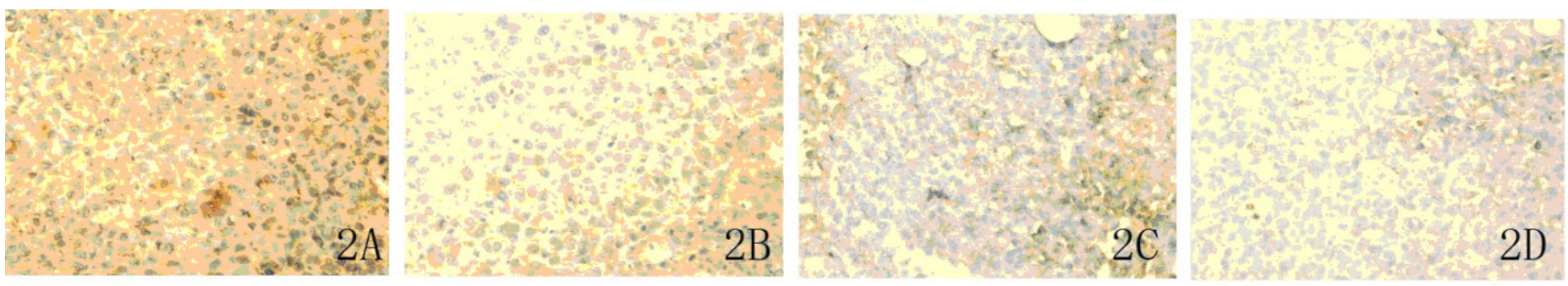

Figure 2. Immunohistochemical staining of CD31. The blood vessels in tumor tissues are stained with yellow brown. A: Control group; B: ginsenoside Rg3 group; C: CPA group; D: ginsenoside Rg3 plus CPA group (magnification ×200).

approach for the treatment of lung cancer. The rationale, as set forth by Folkman and others (Fukushima, 1983), states that establishing a state of counterbalancing between apoptosis and tumor cell proliferation by inhibiting angiogenesis induced by tumor and thus depriving tumors of essential nutrients and oxygen, leading to a quiescent state in which tumor expansion is stalled (Ellis et al., 2001). Ginsenoside Rg3, the major active component of ginseng, exerts angiosuppressive effects in numerous cancers (Yue et al., 2006; Chen et al., 2005). In this study, the results show that ginsenoside Rg3 inhibited the tumor growth and angiogenesis in lung cancer. Studies have confirmed that VEGF played an important role in the angiogenesis, and its expression was correlated with MVD (Maria et al., 2005). Our study discloses that ginsenoside $\mathrm{Rg} 3$ significantly 
Table 3. Inhibitory effect of ginsenoside Rg3 and CPA on expression of VEGF.

\begin{tabular}{|c|c|c|c|c|c|c|}
\hline \multirow{2}{*}{ Group } & \multirow{2}{*}{ Number of mice } & \multicolumn{4}{|c|}{ VEGF Expression } & \multirow{2}{*}{$P$ value } \\
\hline & & - & + & ++ & +++ & \\
\hline Control & 7 & 0 & 0 & 1 & 6 & \\
\hline Rg3 & 7 & 2 & 4 & 1 & 0 & $<0.05^{*}$ \\
\hline CPA & 7 & 2 & 4 & 0 & 1 & $<0.05^{*}$ \\
\hline $\mathrm{CPA}+\mathrm{Rg} 3$ & 7 & 1 & 5 & 1 & 0 & $<0.05^{*}$ \\
\hline
\end{tabular}

${ }^{*} P$ values vs. control group. VEGF, vascular endothelial cell growth factor
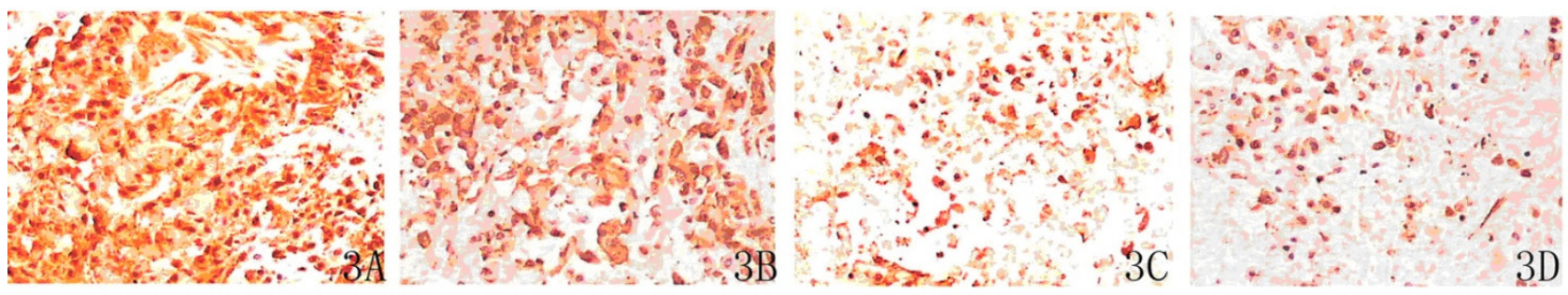

Figure 3. Immunohistochemical staining of VEGF. VEGF positive cells have yellow brown granules in the cytoplasm. A: Control group; B: ginsenoside Rg3 group; C: CPA group; D: ginsenoside Rg3 plus CPA group (magnification $\times 200$ ).

decreased the expression of VEGF in lung cancer. These findings indicate that ginsenoside Rg3 could inhibit the tumor growth through its angiosuppressive effects. To prove the anti-cancer effects of ginsenoside Rg3, we assess the proliferative activity of lung cancer cells through PCNALI. PCNA is the polymerase $\delta$ accessory protein and synthesized in the early $\mathrm{G} 1$ and $\mathrm{S}$ phases of cell cycle when DNA synthesis is dominant. Therefore, PCNA can reflect the DNA replication. Furthermore, the metabolism and DNA replication in tumor cells represent the proliferative activity. Thus, PCNA is frequently used as an indicator of proliferative activity of tumor cells (Isozaki et al., 1996; Niu et al., 2002). Ginsenoside Rg3 inhibits the PCNA expression that causes the reduced proliferative activity of lung cancer. Based on these results, we postulate that ginsenoside Rg3 may be an effective inhibitor of angiogenesis. However, in contrast to the data generated from animal studies, the previous results of various clinical trials aiming at evaluating angiogenic inhibitors in cancer therapy have been equivocal. A strategy combining continuous low dose chemotherapy and anti-angiogenic therapy has been developed recently and used in the experimental treatment of malignant tumors such as breast cancer, retinoblastoma, lymphoma and leukemia (Overmoyer et al., 2007; Bello et al., 2001; Morioka et al., 2003). The combination of low dose chemotherapy and anti-angiogenesis conferred more effective anti-cancer effects than conventional chemotherapy. This study evaluates the effectiveness of the combination of anti-angiogenic therapy and chemotherapy in the treatment of lung cancer. The results show that the combination of ginsenoside $\mathrm{Rg} 3$ and CPA prolonged the survival time of mice with lung cancer when compared with the treatment with CPA or ginsenoside Rg3 alone. Therefore, the combination of ginsenoside Rg3 and CPA has advantages in anti-cancer over ginsenoside $\mathrm{Rg} 3$ or CPA alone. Additionally, ginsenoside $\mathrm{Rg} 3$ and CPA exerted synergistic angiosuppressive effects confirm by the decreased MVD and VEGF expression. All these findings aforementioned demonstrate that the combination of ginsenoside $\mathrm{Rg} 3$ and CPA was more effective in suppressing cancer growth than conventional therapy.

\section{Conclusions}

Taken together, this study suggests that ginsenoside $\mathrm{Rg} 3$, alone or in combination with CPA inhibits cancer growth and angiogenesis in lung cancer. Furthermore, the combination of ginsenoside Rg3 and CPA confers synergistic angiosuppressive effects and improves the quality of life of mice with lung cancer. The combination of chemotherapy and antiangiogenic therapy may be a promising candidate in the experimental treatment of lung cancer.

\section{REFERENCES}

Bello L, Carrabba G, Giussani C, Lucini V, Cerutti F, Scaglione F (2001). Low dose chemotherapy combined with an antiangiogenic drug reduces human glioma growth in vivo. Cancer Res., 61(20): 
7501-7506.

Chen MW, Ni L, Zhao XG, Niu XY (2005). The inhibition of 20(R)ginsenoside $\mathrm{Rg} 3$ on the expressions of angiogenesis factors proteins in human lung adenocarcinoma cell line A549 and HUVEC304 cell (Chin). Chin. J. Chin. Materia Medica, 30(5): 357-360.

Davis K, Yount S, Wagner L, Cella D (2004). Measurement and management of health-related quality of life in lung cancer. Clin. Adv. Hematol. Oncol., 2(8): 533-40.

Ellis LM, Liu W, Ahmad SA, Fan F, Jung YD, Shaheen RM (2001). Overview of angiogenesis: biologic implications for antiangiogenic therapy. Semin Oncol. 28(5): 94-104.

Hanahan D, Bergers G, Bergsland E (2000). Less is more, regularly: metronomic dosing of cytotoxic drugs can target tumor angiogenesis in mice. J. Clin. Invest., 105(8): 1045-1047.

Isozaki H, Okajima K, Ichinona T (1996). Significance of proliferating cell nuclear antigen (PCNA) expression in gastric cancer in relation to lymph node metastasis. J. Surg. Oncol., 61(2): 106-110.

Keum YS, Han SS, Chun KS, Park KK, Park JH, Lee SK (2003). Inhibitory effects of the ginsenoside Rg3 on phorbol ester-induced cyclooxygenase-2 expression, NF-kappaB activation and tumor promotion. Mutat. Res., 523-524: 75-85.

Kim HS, Lee EH, Ko SR, Choi KJ, Park JH (2004). Effects of ginsenosides Rg3 and Rh2 on the proliferation of prostate cancer cells. Arch. Pharm. Res., 27(4): 429-435.

Klement G, Baruchel S, Rak J, Man S, Clark K, Hicklin DJ (2000). Continuous low dose therapy with vinblastine and VEGF receptor-2 antibody induces sustained tumor regression without overt toxicity. J. Clin. Invest., 105(8): 1-17.

lishi H, Tatsuta M, Baba M, Uehara H, Nakaizumi A, Shinkai K (1997). Inhibition by ginsenoside $\mathrm{Rg} 3$ of bombesin-enhanced peritoneal metastasis of intestinal adenocarcinomas induced by azoxymethane in Wistar rats. Clin. Exp. Metastasis, 15(6): 603-611.

Lee SY, Kim GT, Roh SH, Song JS, Kim HJ, Hong SS (2009). Proteomic analysis of the anti-cancer effect of 20S-ginsenoside Rg3 in human colon cancer cell lines. Biosci Biotechnol Biochem. 73(4): 811-816.
Liu Jw, Chen JX, Yu LH, Tian YX, Cui XY, Yan Q (2004). Inhibitory effect of ginsenoside-Rg3 on lung metastasis of mouse melanoma transfected with ribonuclease inhibitor (Chin). Chin. J. Oncol., 26(12): 722-725.

Maria RR, Francesca C, Francesca G (2005). Microdensity vessels and with vascular endothelial growth factor expression in ovarian carcinoma. Int. J. Surg. Pathol., 13(87): 135-142.

Mochizuki M, Yoo YC, Matsuzawa K, Sato K, Saili I, Tonooka S (1995). Inhibitory effect of tumor metastasis in mice by saponin, ginsenoside$\mathrm{Rb2}, 20(\mathrm{R})$ - and 20(S)-ginsenoside- Rg3, of red ginseng. Biol. Pharm. Bull., 18(5): 1197-202.

Morioka H, Weissbach L, Vogel T, Petur NG, Faircloth GT, Shao L (2003). Antiangiogenesis treatment combined with chemotherapy produces chondrosarcoma necrosis. Cancer Res. 9(3): 1211-17.

Nguyen JT, Wu P, Clouse ME, Hlatky L, Terwillinger EF (1998). Adenoassociated virus-mediated delivery of antiangiogenic factors as an antitumor strategy. Cancer Res. 58(24): 5673-5677.

Niu YJ, Liu FL, Zhou ZY, Wang HY (2002). Expression of CD44V6 and PCNA in squamous cell carcinomas. Chin. Med. J., 115(10): 15641568.

Overmoyer B, Fu P, Hoppel C, Radivoyevitch T, Shenk R, Persons M (2007). Inflammatory breast cancer as a model disease to study tumor angiogenesis: results of a phase IB trial of combination SU5416 and doxorubicin. Clin. Cancer Res., 13(19): 5862-5868.

Yue PY, Wong DY, Wu PK, Leung PY, Mak NK, Yeung HW(2006). The angiosuppressive effects of 20(R)-ginsenoside Rg3. Biochem. Pharmacol., 72(4): 437-445.

Zhang Q, Kang X, Yang B, Wang J, Yang F ( 2008 ). Antiangiogenic effect of capecitabine combined with ginsenoside Rg3 on breast cancer in mice. Cancer Biother Radiopharm. 23(5): 647-653. 